\title{
GAME THEORETIC ASPECTS OF CROWD RENTING.
}

\author{
NWOBI-OKOYE, C.C. \\ Anambra State University, Uli, Anambra State, Nigeria \\ nwobi@ansu.edu.ng, chidozien@yahoo.com
}

\begin{abstract}
Politicians and businessmen use various strategies to outwit their opponents. One such strategy is crowd renting, a powerful virtual strategy. A mathematical model developed by Nwobi-Okoye using game theory is used to show that crowd renting would always be the equilibrium strategy in strategic interactions involving political actors and businesses, provided the cost of using such strategies does not exceed a certain critical threshold where using it would no longer be the equilibrium strategy. It is equally shown that crowd renting would improve the electoral/business fortunes of parties/candidates/businesses and queuing system performance by increasing the number of possible voters or customers as the case may be. Finally it is shown mathematically that delaying voting in opposition strongholds or delaying the start of business by an opponent makes the opposition or opponent less likely to win.
\end{abstract}

Keywords: Crowd renting; Game theory; Equilibrium; Virtual strategy

\section{INTRODUCTION}

In a previous work of the author where the concept of virtual reality in game theory was introduced, It was noted that crowd renting, an important strategy in politics and business, could be regarded as a virtual strategy (Nwobi-Okoye, 2009). Political actors and businessmen have used such strategies over the years with good measure of success to out maneuver their opponents.

Exploiting the crowd effect has been one of the tools in the arsenal of political actors over the years. The late Nigerian military head of state, General Sani Abacha, used it in his famous 2 million man match held in the Nigerian capital, Abuja in the year 1998. The match was organized at the height of his struggle to succeed himself as the civilian Head of State. Insiders knew the crowd used during the match was actually rented to give the impression that Nigerians were solidly in support of his plan to transmute into a civilian leader which his administration was then organizing. In Nigeria, crowd renting has been an accepted part of the electioneering process for a very long time, and rented crowds at political rallies is not uncommon.

In most countries in Africa, crowd renting tactics by unpopular leaders desperate to hang on to power abound (Muchemwa, 2010), (Okumu, 2010). Quite often, such tactics worked well for such leaders, as in the case of Zimbabwe where President Robert Mugabe used it effectively to out maneuver his opponents.

Another facet of crowd renting is widely used in commercial motor parks in Nigeria where passengers board vehicles to their various destinations. In this situation, dummy passengers are used in commercial vehicles to induce passengers to board them. This has been used with great success for many years in Nigerian motor parks. Some business organizations like banks adopt this strategy where the actual number of persons in a queue is deliberately hidden to induce more persons to join the queue.

In the light of the above, crowd renting was studied scientifically in order to understand better the mechanisms of its occurrence so that political actors and business strategists can use it more effectively. One possible salient scientific method of studying crowd renting is through the application of game theoretic modeling to understand the scientific basis to crowd renting successes.
This paper presents the results of applying game theoretic model developed by Nwobi-Okoye (2009) to analyze crowd renting.

\section{THEORETICAL BACKGROUND}

Crowd Renting and Virtual Reality: A crowd is a large and definable group of people. A crowd may be definable through a common purpose or set of emotions, such as at a political rally, at a sports event, or simply be made up of many people going about their business in a busy area (e. g. shopping). Crowd psychology is a branch of social psychology. The psychological aspects of crowd are concerned with the psychology of the crowd as a group and the psychology of those who allow their will and emotions to be informed by the crowd. Ordinary people can typically gain direct power by acting collectively. Historically, because large groups of people have been able to bring about dramatic and sudden social change in a manner that bypasses established due process, they have also provoked controversy (Wikipedia, 2010).

Social scientists have developed several different theories for explaining crowd psychology, and the ways in which the psychology of the crowd differs significantly from the psychology of those individuals within it (Wikipedia, 2010). One notable theorist on crowd psychology is Sigmund Freud. The main idea of Sigmund Freud's crowd behaviour theory is that people who are in a crowd act differently towards people from those who are thinking individually. In other words, opinions of individuals in a crowd are the crowd's opinion. The minds of the group would merge to form a way of thinking. Each member's enthusiasm would be increased as a result, and one becomes less aware of the true nature of one's actions (Jones, 1958; Tauber, 2010). The corner stone of this paper is based on Freud's theory of crowd.

Virtual reality occurs when the payoff determining factors assume certain conditions exist which in fact do not. Virtual reality strategies use deceptive perceptions used to improve payoffs for the strategist (Nwobi-Okoye, 2009).

When the crowd in a political rally is rented, intending voters apparently unaware of this have a tendency to follow the crowd. Similarly, if the crowd in the commercial vehicle being loaded is rented, intending passengers apparently unaware of this tends to join the vehicle since they assume the vehicle is about to be full to commence the journey. This is virtual reality because in the first example, the crowd does not support the given political party; it is just the voter's perception. Parties/candidates that have rented crowd are thus more likely to attract more voters than parties/candidates that have not rented their crowd.

\section{THE MODEL}

The game theoretic model used to model crowd renting is the Markov queue game model (Nwobi-Okoye, 2009). The following assumptions were made for the purpose of the modeling:

Game Characteristics and Assumptions:The mathematical model for analyzing crowd renting was developed based on the following game characteristics and assumptions:

1. The game corresponds to the game developed by NwobiOkoye (2009).

2. An infinite population source which represents possible voters/customers is assumed. 
3. Crowd renting increases the probability of a voter/customer queuing behind a particular candidate or business.

4. Crowd renting improves the overall performance of the queuing system in terms of the number served.

Mathematical Analysis: The mathematical analysis is based on the assumption that two candidates in an election try to out-campaign each other by using crowd renting as a campaign strategy, or that two businesses try to make more gains and out perform each other by adopting crowd renting strategies. Each voter/customer could queue behind (vote for or patronize) any of the candidates/businesses he/she prefers. The entire mathematical analysis is based on the model developed by Nwobi-Okoye (2009). The applicable equations for modeling the game theoretic aspect of crowd renting are:

$E G=P \bullet G$

The dot operator in equation 1 carries out the operation of multiplying each payoff by its associated probability of occurrence.

$G T=G-(n-s) z$
$G T_{L X J X}=G_{L X J X}-\left(n_{X}-s\right) z$
$G T_{I Y J Y}=G_{I Y J Y}-\left(n_{Y}-s\right) z$

For variant 2 games, equations 2, 3 and 4 are of the form shown in equations 5,6 and 7 :

$G T=G-R$

Here, $\mathrm{R}$ is the payoff reduction.

$\mathrm{R}$ represents the extra cost incurred due to crowd renting.

$$
\begin{aligned}
& G_{I X J X}=G_{I X J X}{ }^{-R} X \\
& G T_{I Y J Y}=G_{I Y J Y}-R_{Y}
\end{aligned}
$$

For variant 1 and 2 games, if crowd renting/hiding is used by any of the players, equation 1 changes to:

$E G=P T \bullet G T$.

The grand payoff matrix, GG, is shown in Fig. 1.
0

\begin{tabular}{c|c}
0 & $G G_{1 X, 1 X}, G G_{1 Y, 1 Y}$ \\
1 & $G G_{2 X, 1 X}, G G_{2 Y, 1 Y}$ \\
$\cdot$ & $\cdot$ \\
$\cdot$ & $\cdot$ \\
$\cdot$ & $\cdot$ \\
$N$ & $G G_{N X, 1 X}, G G_{N Y, 1 Y}$
\end{tabular}
$N$

$G G_{1 X, 1 X}, G G_{1 Y, 1 Y} \quad \ldots G G_{1 X, N X}, G G_{1 Y, N Y}$

$G_{2 X, 2 X}, G G_{2 Y, 2 Y} \quad \ldots G G_{2 X, N X}, G G_{2 Y, N Y}$

$G G_{N X, 2 X}, G G_{N Y, 2 Y}$

$\ldots G G_{N X, N X}, G G_{N Y, N Y}$

FIG. 1. GRAND PAYOFF MATRIX, GG

Here

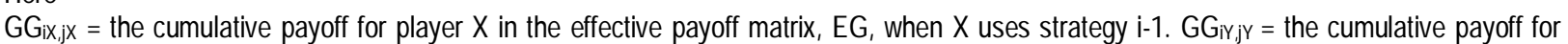
player $Y$ in the effective payoff matrix, EG, when $Y$ uses strategy j-1.

$$
\begin{aligned}
G G_{I Y J Y} & =\sum_{I=1}^{N} \sum_{J=1}^{N} E G_{I Y J Y^{*}} \\
G_{I X J X} & =\sum_{I=1}^{N} \sum_{J=1}^{N} E G_{I X J X^{*}}
\end{aligned}
$$

$\mathrm{N}=$ maximum value of strategies i. e. possible number of crowd renting strategies.

APPLICATION CASE STUDIES

Definitions: The appropriate class of virtual games to be used for the modeling as I have said earlier is the Markov Queue Game. In this game the matrix $\mathrm{P}$ in equation 1 is a Markov transition matrix whose nature is shown in Fig. 2. 
Next State

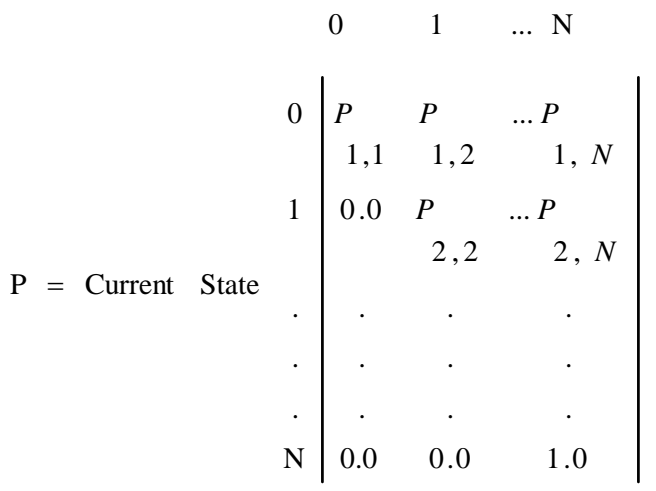

\section{FIG 2. TRANSITION MATRIX FOR EACH COMPETITOR}

Assuming two competitors are denoted by $X$ and $Y$, the unified transition matrix $P U$ obtained by combining the transition matrices of players $X$ and $Y$ denoted by $P x$ and $P y$ respectively is shown in fig. 3 below:

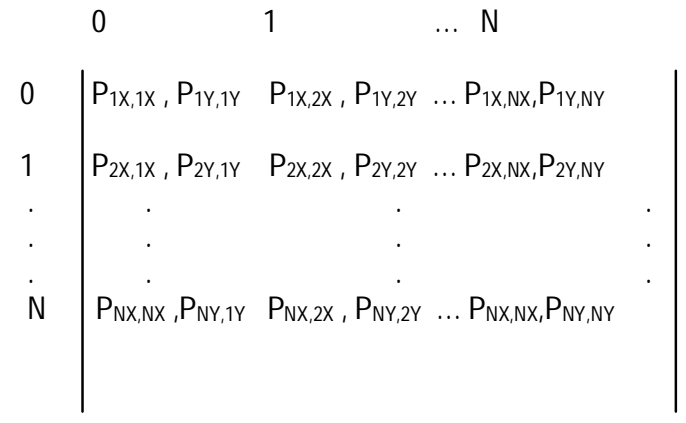

FIG. 3. UNIFIED TRANSITION MATRIX, PU

Let the gain per voter be estimated with the formula:

$$
G P V=\frac{G T}{N}
$$

Where

GPV=Gain per voter

$\mathrm{N}=$ number of possible voters

GT=Total possible Gain

The cost of crowd renting per voter could be estimated with the formula:

$$
C P V=\frac{C}{N}
$$

Where

$\mathrm{CPV}=$ Cost per voter

$\mathrm{N}=$ number of possible voters

$\mathrm{C}=$ cost of crowd renting

Observation 1: If the utility functions for two players $X$ and $Y$ which are denoted by $f(n)$ and $g(n)$ respectively are functions of $n$ where $n$ is the number of voters, if $f(n)>g(n)$ and $C P V$ remain constant, it follows that at equilibrium,

\section{$G_{I X J X}>G G_{I Y I Y}$. Provided the game is not biased in favour of any player.}

Proof: Let GPVx denote gain per voter for player X and GPVy the gain per voter for player $\mathrm{Y}$. 
If $\mathrm{f}(\mathrm{n})>\mathrm{g}(\mathrm{n})$, it follows that $\mathrm{GPV} x>\mathrm{GPV} y$, since from equations 9 and $10 G G_{I Y J Y}=\sum_{I=1}^{N} \sum_{J=1}^{N} E G_{I Y J Y}$ and $G G_{I X J X}=\sum_{I=1}^{N} \sum_{J=1}^{N} E G_{I X J X}$, it follows that at equilibrium $G G_{I X J X}>G G_{I Y I Y}$ provided the game is not biased in favour of any player.

Proposition 1: Let $G G_{I X} J X_{n}$ represent elements of competitor $\mathrm{X}$ of the matrix $\mathrm{GG}$ at period $\mathrm{n}$ and $G G_{I X_{n+1}} J X_{n+1}$ at period $\mathrm{n+1}$. It follows that $G G_{I X} J X_{n}<G G_{I X_{n+1}} J X_{n+1}$ provided the equilibrium of the transition matrix $\mathrm{P}$ is not reached at period $\mathrm{n}$.

Corollary: Similarly, $G G_{I X} J X_{n}=G G_{I X_{n+1}} J X_{n+1}$ provided the equilibrium of the transition matrix $\mathrm{P}$ is reached at period $\mathrm{n}$.

Proof: It can easily be shown that for the transition matrix $\mathrm{P}$, that $\sum_{n=1}^{\infty} P_{i i}^{(n)} \neq \infty$ Provided $\mathrm{i} \neq \mathrm{N}$

Therefore, each state is transient except $\mathrm{N}$. Since state $\mathrm{N}$ is the absorbing state and transition matrix $\mathrm{P}$ is reducible; hence, at equilibrium the probability of staying at state $\mathrm{N}$ is one, while the probability of staying in any other state is zero. From equation $1 \mathrm{EG}=\mathrm{P} \cdot \mathrm{G}$ and from equation

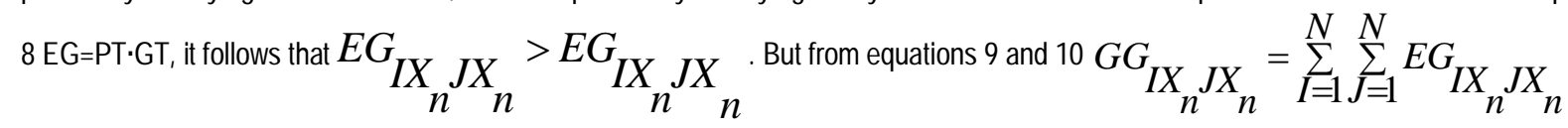
and $G G_{I X_{n+1} J X_{n+1}}=\sum_{I=1}^{N} \sum_{J=1}^{N} E G_{I X_{n+1}} J X_{n+1}$

It follows that provided the equilibrium of the transition Matrix P is not reached, $G G_{I X_{n}} J X_{n}<G G_{I X_{n+1}} J X_{n+1}$.

Proposition 2: For a given payoff reduction factor $z$, if strategy $n$ is optimal $i$. e. an equilibrium at period $n$, it may not necessarily be optimal at period $n+1$ provided the equilibrium state of the transition matrix $P$ is not reached at period $n$ and the game is not biased in favour of any player.

Corollary: For a given payoff reduction factor $z$, if strategy $n$ is optimal i.e. an equilibrium at period $n$, it is also optimal at period $n+1$ provided the equilibrium state of the transition matrix $P$ is reached at period $n$ and the game is not biased in favour of any player.

Proof: Assuming some values of payoff reduction factor z denoted by z1 and z .Let $G G_{I X} J X_{n_{z 1}}$ represent the gain for competitor X while playing the optimal strategy $\mathrm{n}$ at period $\mathrm{n}$ when the payoff reduction factor is $\mathrm{z} 1$ and

${ }^{G} G_{(n+1)} X J X_{(n+1)}$ represent the gain for competitor $\mathrm{X}$ while playing the optimal strategy $\mathrm{n}$ at period $\mathrm{n}+1$

when the payoff reduction factor is $z 2$. Since $z 1=z 2$ and from proposition 1 it is shown that at equilibrium

$G G_{I X_{n} J X_{n}}=G G_{I X_{n+1}} J X_{n+1}$, it follows that for a given payoff reduction factor z, if strategy $\mathrm{n}$ is optimal i. e. an equilibrium at

period $n$, it is also optimal at period $n+1$ provided the equilibrium state of the transition matrix $P$ is reached at period $n$ and the game is not biased in favour of any player.

Proposition3: For variant 1 games, if strategy $n 1$ is used by player $X$ and $n 2$ is used by player $Y$. If $n 1>n 2$, the transition matrix for player $X$ reaches equilibrium before that for player $\mathrm{Y}$ and if $G G_{I X_{k}} J X_{k}$ represent elements of competitor $\mathrm{X}$ of the matrix GG at period k 
and $G G_{I Y_{k} J Y_{k}}$ for competitor $Y$ at period k. It follows that $G G_{I X_{k}} J X_{k}>G G_{I Y_{k}} J Y_{k}$ provided the equilibrium of the transition matrix $\mathrm{P}$ is not reached at period $\mathrm{n}$.

Proof: In matrix PU above if strategy $\mathrm{n} 1$ is used by player $\mathrm{X}$ and $\mathrm{n} 2$ is used by player $\mathrm{Y}$, since $\mathrm{n} 1>\mathrm{n} 2$ virtual strategy is used by player $\mathrm{X}$, hence $P_{I X J X}<P_{I Y J Y}$ where i=0 and j<n and $P_{I X J X}>P_{I Y J Y}$ where i=0 or j>n. It can comfortably be assumed that all other elements of the matrix PU remain the same. But from proposition 1 it is shown that each state of the Transition Matrix $\mathrm{P}$ is transient except $\mathrm{N}$, and state $\mathrm{N}$ is the absorbing state. Since transition matrix $\mathrm{P}$ is reducible, it follows that if the transition matrix for player $\mathrm{X}, \mathrm{Px}$ reaches equilibrium at period $\mathrm{m} 1$ and the transition matrix for player $\mathrm{Y}$, Py reaches equilibrium at $\mathrm{m} 2$ that $\mathrm{m} 1>\mathrm{m} 2$.

Similarly, from equations 9 and 10.

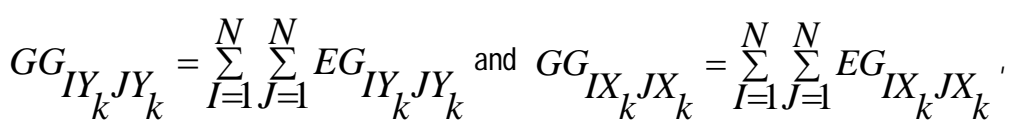

since $\sum_{I=1}^{N} \sum_{J=1}^{N} E G_{I X_{k} J X_{k}}>\sum_{I=1}^{N} \sum_{J=1}^{N} E G_{I Y_{k} J Y_{k}}$, it follows that at equilibrium $G G_{I X_{k} J X_{k}}>G G_{I Y_{k} J Y_{k}}$ provided the game is not biased in favour of any player.

\section{CASE 1: VOTING}

The mathematical model used in this case corresponds to variant 1 games as mentioned above. Here it is assumed that political parties or candidates for an election use crowd renting as campaign strategy during an election.

The voting is assumed to be electronic voting and the queuing system for the voting system could be represented by Fig. 4 .

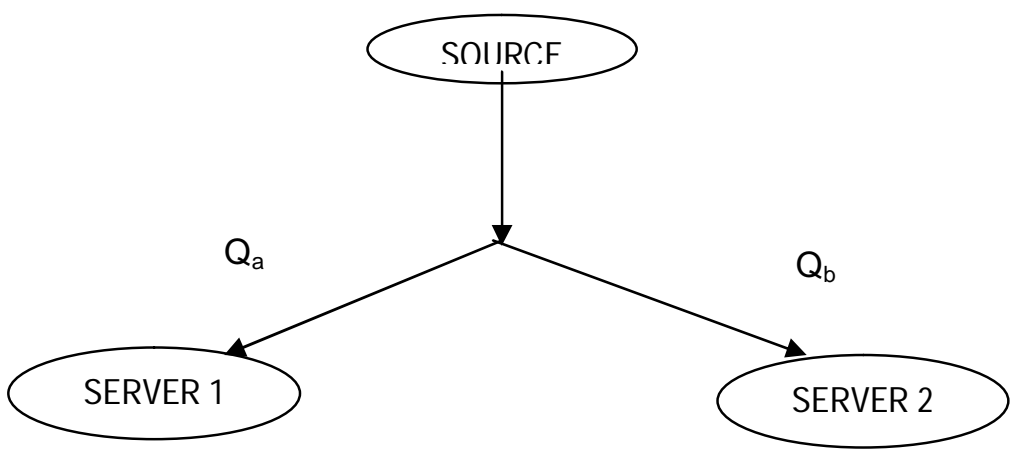

FIG. 4 QUEUING MODEL FOR A VOTING SYSTEM

Here

$\mathrm{Q}_{\mathrm{a}}=$ voter for party/candidate a

$\mathrm{Q}_{b}=$ voter for party/candidate $b$

The servers in Fig. 4 are assumed to have a finite capacity of two. The voting which is electronic voting, is assumed to take place in batches of two, hence a maximum of two voters can cast their votes simultaneously with the electronic system. Voters use different machines depending on the candidate/party they wish to vote for.

Problem Statement: Let us assume that proportional representation is used in an election and that the gain made from each vote is 20 units. Assuming that employing crowd renting strategies takes away either 1unit or 5 units from the gain which reduces the gain to 19 units or 15 units. If we assume an infinite population source and equally assume that the voting is done in batches of two voters per batch.

How could game theoretic modeling be used to determine the equilibrium point assuming two parties/candidates campaign for an election and could either use crowd renting or not?

This problem could be solved by assuming a 2 strategy, 2-persons Markov queue game which will be evaluated shortly.

Evaluation of the 2 Strategy, 2-Persons Game: Typical transition matrices for the two candidates/parties are as follows: 


$$
\begin{aligned}
& \text { Next State } \\
& \begin{array}{lll}
0 & 1 & 2
\end{array}
\end{aligned}
$$

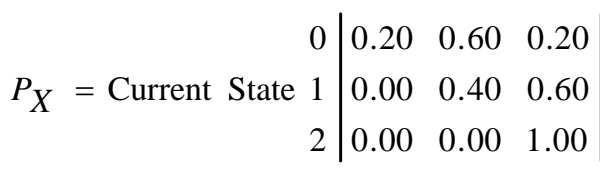

$$
\begin{aligned}
& \begin{array}{lll}
0 & 1 & 2
\end{array}
\end{aligned}
$$

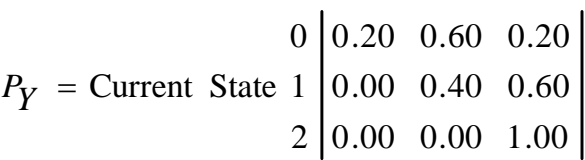

For the above transition matrices, we assumed that the probability of moving from a higher state to a lower state is zero (0).

If the game is biased in favour of $X, P_{x}$ looks like this:

\section{Next State}

$$
\begin{aligned}
& \begin{array}{lll}
0 & 1 & 2
\end{array}
\end{aligned}
$$

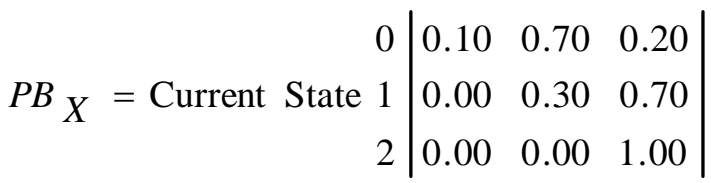

The matrix could be biased in favour of a particular candidate/party for some reasons namely:

- $\quad$ The candidate/party is favoured by the majority ethnic group, religious denomination, age group etc.

- $\quad$ The party/candidate has more resources at his/her disposal.

A unified transition matrix, $\mathrm{P}$, of the game produced by combining $\mathrm{Px}$ and $\mathrm{Py}$ is shown below:

$$
\begin{array}{r|ccc} 
& 0 & 1 & 2 \\
P_{0}=1 & 0.20,0.20 & 0.60,0.60 & 0.20,0.20 \\
2 & 0.00,0.00 & 0.40,0.40 & 0.60,0.60 \\
0.00,0.00 & 0.00,0.00 & 1.00,1.00
\end{array} \mid
$$

A typical payoff matrix $\mathrm{G}_{0}$ for the game is shown below:

$$
G_{0}=\left|\begin{array}{ccc}
0,0 & 20,20 & 40,40 \\
0,0 & 0,0 & 20,20 \\
0,0 & 0,0 & 0,0
\end{array}\right|
$$

The effective payoff matrix for the game, $E_{0}$, is given by: $E G_{0}=G_{0} \cdot P_{0}$

$$
E G_{0}=\left|\begin{array}{ccc}
0.0,0.0 & 12.0,12.0 & 8.0,8.0 \\
0.0,0.0 & 0.0,0.0 & 12.0,12.0 \\
0.0,0.0 & 0.0,0.0 & 0.0,0.0
\end{array}\right|
$$

Possible total payoff for player $X=32.0$

Possible total payoff for player $Y=32.0$

The transition and payoff matrices above represent the natural state of the game.

Since crowd renting is virtual reality, introducing it as a strategy would, as expected, improve the payoff of the competitors using the strategy 
(Nwobi-Okoye, 2009). Hence, a unified transition matrix of the game produced by combining matrix Px and Py is assumed to change to PT as shown below, in accordance with virtual strategy theorem (Nwobi-Okoye, 2010).

$$
P T=\left|\begin{array}{lll}
0.0,0.0 & 0.7,0.0 & 0.3,0.0 \\
0.0,0.7 & 0.2,0.2 & 0.8,0.0 \\
0.0,0.3 & 0.0,0.8 & 1.0,1.0
\end{array}\right|
$$

For the payoff matrix $G_{0}$ above, if strategy 1 which is virtual strategy is used and $R=1.0$, we have from equation 2 :

$$
\begin{gathered}
G T=\left|\begin{array}{ccc}
-1.0,-1.0 & 19.0,19.0 & 38.0,38.0 \\
0.0,0.0 & 0.0,0.0 & 19.0,19.0 \\
0.0,0.0 & 0.0,0.0 & 0.0,0.0
\end{array}\right| \\
P T=\left|\begin{array}{lll}
0.0,0.0 & 0.7,0.0 & 0.3,0.0 \\
0.0,0.7 & 0.2,0.2 & 0.8,0.0 \\
0.0,0.3 & 0.0,0.8 & 1.0,1.0
\end{array}\right|
\end{gathered}
$$

The effective payoff matrix, $\mathrm{EG}_{1}$, is given by: $\mathrm{EG}=\mathrm{GT} \cdot \mathrm{PT}$

Total payoff for player $X=37.7$

$$
E G_{1}=\left|\begin{array}{ccc}
0.0,0.0 & 13.3,13.3 & 11.4,11.4 \\
0.0,0.0 & -0.2,-0.2 & 15.2,15.2 \\
0.0,0.0 & 0.0,0.0 & 0.0,0.0
\end{array}\right|
$$

Total payoff for player $Y=37.7$

The grand payoff matrix, GG for $R=1.0$ is given by:

$$
G G=\begin{aligned}
& 0 \\
& 1
\end{aligned}\left|\begin{array}{ll}
32.0,32 \cdot 0 & 32.0,37.7 \\
37.7,32 \cdot 0 & 37.7,37.7
\end{array}\right|
$$

A look at the payoff matrix, GG, above shows that nash equilibrium point corresponds to strategy 1,1 .

For the payoff matrix $G_{0}$ above, if strategy 1 which is virtual strategy is used and $R=5.0$, we have from equation 2 :

$$
\begin{gathered}
G T=\left|\begin{array}{ccc}
-5.0,-5.0 & 15.0,15.0 & 30.0,30.0 \\
0.0,0.0 & 0.0,0.0 & 15.0,15.0 \\
0.0,0.0 & 0.0,0.0 & 0.0,0.0
\end{array}\right| \\
P T=\left|\begin{array}{lll}
0.0,0.0 & 0.7,0.0 & 0.3,0.0 \\
0.0,0.7 & 0.2,0.2 & 0.8,0.0 \\
0.0,0.3 & 0.0,0.8 & 1.0,1.0
\end{array}\right|
\end{gathered}
$$

The effective payoff matrix, $\mathrm{EG}_{1}$, is given by: $\mathrm{EG}_{1}=\mathrm{GT} \cdot \mathrm{PT}$

$$
E G_{1}=\left|\begin{array}{ccc}
0.0,0.0 & 10.5,10.5 & 9.0,9.0 \\
0.0,0.0 & -0.1,-0.1 & 12.0,12.0 \\
0.0,0.0 & 0.0,0.0 & 0.0,0.0
\end{array}\right|
$$

Total payoff for player $X=37.7$

Total payoff for player $Y=37.7$ 
The grand payoff matrix, $G G$ for $R=5.0$ is given by:

$$
G G=\begin{array}{l|ll|}
0 & 32.0,32.0 & 32.0,30.5 \\
1 & 30.5,30.5 & 30.5,30.5
\end{array} \mid
$$

A look at the payoff matrix, GG, above shows that nash equilibrium (Nash, 1950) point corresponds to strategy 0, 0.

CASE 2: BUSINESS PATRONAGE: In previous mathematical analysis (Nwobi-Okoye, 2009), variant 1 model was used in input based crowd renting system. A practical example was the system earlier mentioned where dummy passengers are used in commercial vehicles to induce passengers to board them, or in situations where the actual number of persons in a queue are hidden from would be customers.

The queuing model in this case is similar to that of variant 1 as shown in Fig. 4. But in this case:

$\mathrm{Q}_{\mathrm{a}}=$ Customer for business a

$\mathrm{Q}_{b}=$ Customer for business $\mathrm{b}$

The servers in Fig. 4 are assumed to have a finite capacity. The service is assumed to take place simultaneously in batches; hence, if the server capacity $\mathrm{N}$ is two, a maximum of two customers can be served simultaneously by the server. Customers queue behind each business/player depending on their preferences. This scenario is similar to what happens in motor parks in Nigeria, where the commercial vehicles have a finite capacity which represents the number of passengers it could accommodate. The customers are served simultaneously because the passengers leave the park simultaneously when the vehicle is full.

\section{RESULTS AND DISCUSSION}

From the evaluation above it could be deduced that the application of virtual strategies (crowd renting) increase the possibility of either party/candidate gaining a vote. But application of virtual strategy comes at a price, hence at a certain cost the virtual strategy becomes optimal for either player. But as the cost increases, non virtual strategy becomes optimal. Hence, there must always be a complete economic analysis before applying virtual strategies in Markov Queue Games. The overall effect of crowd renting is that it could improve the electoral fortunes of the parties or candidates. This could be explained from the fact the voter population is assumed to be infinite and crowd renting has the tendency to get more voters out to vote. This phenomenon is similar to advertising which could make more buyers to buy competing products, thereby improving the fortunes of the competing firms.

The consequence of proposition 2 is that if voting in an opponent's stronghold is delayed or if an opponent starts the game later, the chance of the opponent winning is reduced. This explains the basis for the strategy sometimes used by ruling parties which deliberately delays voting in opposition strongholds by delaying the delivery of electoral materials to the strongholds. Of course the period for voting is finite and when voting ends, the game is up.

Propositions 1, 2 and 3 apply to all facets of crowd renting or hiding, whether input based on non input based. Hence, crowd renting/hiding generally improves the performance of a queuing system. Secondly, a competitor that adopts crowd renting/hiding strategy is more likely to win. Thirdly, a player that starts the game earlier than his opponent is more likely to win, a direct consequence of proposition 2.

\section{CONCLUSIONS}

Crowd renting alters the perception of would be customers/voters and improves their possibility of joining the crowd. This is a powerful virtual strategy which should be exploited to the maximum by businessmen, politicians and political actors. The mathematical/analytical basis for its successful exploitation has been laid down in this work.

The analysis done in this work will be very useful to game theorists, political scientists, systems scientists and operations researchers who advise businesses, politicians and political actors.

\section{REFERENCES}

Asmussen, S. (2003). Applied Probability and Queues. Springer Publications, New York.

Fudenberg, D. \& Tirole, J. (1991). Game Theory. MIT Press, Cambridge, MA.

Hamdy, T. (2004). Operations Research. Prentice Hall of India Ltd, New Delhi.

Jones, E. (1958). The Life and Work of Sigmund Freud, 3 vols, Basic Books, USA.

Le Bon, G. (1895). "The Crowd: A Study of the Popular Mind". Available from: http://onlinebooks.library.upenn.edu/webbin/gutbook/lookup?num=445 [Accessed February 23 2010].

Muchemwa, F. (2010). Why African Leaders Don't Give up Power. Available from: http://africanhistory.suite101.com/article.cfm/why_african_leaders_dont_give_up_power [Accessed May 10 2010]

Nash, J. (1950). Equilibrium Points in n-Person Games. National Academy of Sciences of the USA 36:48-49.

Nwobi-Okoye C. (2009). Markovian Queue Game with Virtual Reality Strategies.Science World Journal, 4(3):35-40. 
Science World Journal Vol 5 (No 2) 2010

unw.scienceworldjournal.org

ISSN 1597-6343

Nwobi-Okoye, C. (2010). General Theory of Games with Virtual Strategies. Pacific Journal of Science and Technology. (In press).

Okumu, W. (2010). The Travails and Antics of Africa's "Big Men" - How Power Has Corrupted African Leaders. http://www.theperspective.org/africabigmen.html [Accessed May 10 2010]

Tauber, A. (2010). Freud, the Reluctant Philosopher. Princeton University Press, Princeton, USA.2010.

Wikipedia, (2010). Crowd Psychology. http://en.wikipedia.org/wiki/Crowd psychology [Accessed January 23 2010] 